\title{
Exact solutions of the vorticity equation on the sphere as a manifold
}

\author{
ISMAEL PÉREZ-GARCÍA \\ Centro de Ciencias de la Atmósfera, Universidad Nacional Autónoma de México, Circuito de la Investigación \\ Cientifica s/n, Ciudad Universitaria, 04510 México, D.F. \\ E-mail: ismael@unam.mx
}

Received November 7, 2014; accepted June 1, 2015

\begin{abstract}
RESUMEN
El propósito de este trabajo es representar las soluciones exactas de la ecuación de vorticidad barotrópica sobre la esfera unitaria $S^{2}$ en rotación como una variedad, que son flujos zonales, ondas Rossby-Haurwitz y soluciones generalizadas llamadas modones. Se relacionan los métodos modernos de la teoría de funciones con la esfera definida como una variedad compacta y diferenciable. Cuando ésta se ha comprendido de forma correcta, se esclarece la noción abstracta de mapa local, cambio de mapa y atlas. Uno de los objetivos de este trabajo es entender mejor la solución de la ecuación de vorticidad barotrópica sobre la variedad $S^{2}$ y su utilidad para identificar las propiedades de las soluciones en la variedad Riemanniana $\left(S^{2}, g\right)$. Por lo tanto, estará disponible un tipo más general de espacio que también puede contener información geométrica y analítica sustancial sobre las soluciones a la ecuación de vorticidad barotrópica.
\end{abstract}

\begin{abstract}
The purpose of this paper is to represent the exact solutions of the barotropic vorticity equations (BVE) on the rotating unit sphere $S^{2}$ as a manifold, which are zonal flows, Rossby-Haurwitz waves and generalized solutions named modons. Modern methods of the function theory are connected to the sphere defined as a compact differentiable manifold. When the differentiable manifold $S^{2}$ is well understood, the abstract notion of local chart, change of chart, and atlases becomes evident. One of the aims of this paper is to better understand the solution of the barotropic vorticity equation on the manifold $S^{2}$ and its usefulness to identify the properties of the solutions on the Riemannian manifold $\left(S^{2}, g\right)$. Therefore, a more general type of space will be available, which can also contain substantial geometric and analytic information about solutions for the barotropic vorticity equation.
\end{abstract}

Keywords: Rossby-Haurwitz waves, modons, hydrodynamics equation on manifolds, unit sphere, mathematical analysis of barotropic model.

\section{Introduction}

Let $S^{2}=\left\{x \in R^{3}:|x|=1\right\}$ denote the unit sphere in $R^{3}$. The large-scale dynamics of the atmosphere on the rotating sphere $S^{2}$ can approximately be governed by the non-linear barotropic vorticity equation (BVE), which can be written in the non-dimensional form as:

$\frac{\partial \Delta \Psi}{\partial t}+J(\Psi, \Delta \Psi+2 \mu)=0$ where $\Psi(\lambda, \mu)$ denotes the stream function, $\mu=\sin$ $\phi=\cos \theta,-\pi \leq \lambda \leq \pi,-\frac{\pi}{2} \leq \phi \leq \frac{\pi}{2}, 0<\theta<\pi, \lambda$ the longitude, $\phi$ the latitude, and $\theta$ the colatitude. $\Delta$ is the Laplace-Beltrami operator on a sphere and $J(\Psi, h)$ is the Jacobian.

The following is a solution for Eq. (1) on the sphere proposed by Thompson (1982):

$\Psi(\lambda, \mu, t)=Y_{\nu}\left(\lambda^{\prime}, \mu^{\prime}\right)-\omega \mu+D$ 
where $\left(\lambda^{\prime}, \mu^{\prime}\right)$ are the spherical coordinates relative to a rotated pole $N^{\prime}$ with coordinates $\left(\lambda_{0}, \mu_{0}\right)$ with respect to the original system, and $Y_{\nu}$ is an eigenfunction of the operator Laplace-Beltrami with eigenvalue $\chi_{\nu}$. Verkley(1984) generalized Thompson's solution and demonstrated that $Y_{\nu}$ could be a set of eigenfunctions that contains more than only spherical harmonics. Then Eq. (2) describes a configuration in which the structure $Y_{\nu}$ moves through the zonal flow $-\omega \mu$ with constant velocity $c_{\nu}$ and without changing size and shape. The pole of the primed system $N^{\prime}$ that moves along a latitude at a constant angular velocity $c_{\nu}$ is given by

$c_{\nu}=\omega-\frac{2(\omega+1)}{\chi_{\nu}}$

where $\chi_{\nu}$ is an eigenvalue for the spectral problem $\Delta Y_{\nu}=-\chi_{\nu} Y_{\nu}$. In particular, for spherical harmonics $Y\left(\lambda^{\prime}, \mu^{\prime}\right)$ of degree $n$ corresponding to the eigenvalue $\chi_{\nu}=\chi_{n}=n(n+1)$, Eq. (2) is a Rossby-Haurwitz (RH) wave. RH waves have proven to be very useful to describe the large-scale wave structure of atmospheric circulation in middle latitudes (Rossby, 1939; Haurwitz, 1940). The solution modon is constructed to divide the sphere $S^{2}$ into two regions (Tribbia, 1984; Verkley, 1984, 1987, 1990; Neven, 1992): an inner region $S_{i}$ centered around the pole $N^{\prime}$, and an outer region $S_{o}$ separated from the inner region by a boundary circle in which $\Psi, q$ and its normal derivative $\Psi^{\prime}$ are continuous. Modons are considered appropriate to describe some types of atmospheric blocking events (Verkley, 1990).

Hydrodynamic equations on manifolds were studied by Ebin and Marsden (1970), Szeptycki (1973a, b), Avez and Bamberger (1978), Ghidaglia et al. (1988), Temam (1987) and Ilyin (1993). The existence, unicity and regularity of the solution for the evolution equation (Eq. (1)) on $S^{2}$ were proven by Szeptycki (1973a, b), Avez and Bamberger (1978), Ilyin (1993) and Skiba (2012). Ebin and Marsden (1970) dealt with the motion of an incompressible fluid on manifolds under a differential geometric point of view. Problems from the transition map between the charts are transferred to those of finding geodesics on the group of all volume-preserving diffeomorphisms, to which the methods of global analysis and infinite-dimensional geometry can be applied.

In this paper we study the manifolds $S^{2}$ in terms of the stream function $\Psi$ for an RH wave which is sufficiently smooth and for Wu-Verkley waves and modons which are weakly differentiable of higher orders. Section 2 deals with the compact differentiable manifold $S^{2}$ and the way in which functions are constructed on this manifold. Section 3 shows the types of solutions that will be considered. Another aim of this paper is to deepen the understanding of the BVE solution on the manifold $S^{2}$ and its usage for deriving the properties of solutions to the manifold $\left(S^{2}, g\right)$. The paper concludes with a summary in section 4 .

\section{Structure of functions on the manifold $S^{2}$}

In this section we review some basic facts concerning to the manifold $S^{2}$. We should recall that the unit sphere $S^{2}$ is a compact and connected differentiable manifold. Indeed, because $S^{2}$ is compact it is not possible to cover it with only one chart. A chart of $S^{2}$ is then a pair $(\Omega, \varphi)$ where $\Omega$ is an open subset of $S^{2}$, and $\varphi$ is a homeomorphism of $\Omega$ onto some open subset of $R^{2}$. Let us consider the two charts $\left\{\left(\Omega_{\nu}, \varphi_{\iota}\right)\right.$, $\left.\left(\Omega_{\kappa}, \varphi_{k}\right)\right\}$ of class $C^{p}$ for $S^{2}$ where every chart corresponds to a geographical coordinate group. It is possible to define a coordinate chart that covers most of $S^{2}$ by using the standard spherical coordinate map. Let $\varphi_{\iota}$ denote the coordinate function, which maps from $\left(x_{1}, x_{2}, x_{3}\right)$ to angles $(\lambda, \theta)$ or to $(\lambda, \mu)$. The domain of $\varphi_{l}^{-1}$ is the open set defined by $\lambda \in(-\pi, \pi)$ and $\theta \in(0, \pi)$ (this excludes the poles). The inverse map $\varphi_{\iota}^{-1}$ yields the parameterization $x_{1}=\cos \lambda \sin \theta, x_{2}=\sin \lambda \sin \theta, x_{3}=\cos \theta$ and its variation $\varphi_{\kappa}^{-1}$ yields the parameterization $\varphi_{\kappa}^{-1}$ $\left(\lambda^{\prime}, \theta^{\prime}\right)=\left(\cos \lambda^{\prime} \sin \theta^{\prime}, \cos \theta^{\prime}, \sin \lambda^{\prime} \sin \theta^{\prime}\right)$. The domain of $\varphi_{\kappa}^{-1}$ in the open set defined by $\lambda^{\prime} \in$ $(-\pi, \pi)$ and $\theta^{\prime} \in(0, \pi)$. The charts $\left(\Omega_{\iota}, \varphi_{\iota}\right)$ and $\left(\Omega_{\kappa}, \varphi_{\kappa}\right)$ correspond to poles $\mathrm{N}$ and $\mathrm{N}^{\prime}$ on the sphere $S^{2}$. $\mathrm{N}^{\prime}$ might be taken as the point $\left(\lambda_{0}=-\frac{\pi}{2}\right.$, $\left.\phi_{0}=0\right)$ in the old system and as the angle $\lambda^{\prime}$ in this new north pole, so that the new international date line is the half circle $\Gamma_{\kappa}=\left\{p \in S^{2}:-\frac{\pi}{2}<\lambda(p)<\frac{\pi}{2}, \theta=\frac{\pi}{2},\right\}$ of the old equator in the $x_{1} x_{2}$-plane, on the front where $x_{1} \geq 0$ (Richtmyer, 1981; Skiba, 1989; PérezGarcía, 2001). The international date line, for the chart $\left(\Omega_{\iota}, \varphi_{\iota}\right)$ is the half circle $\Gamma_{\iota}=\left\{p \in S^{2}:-\frac{\pi}{2}<\right.$ $\left.\phi(p)<\frac{\pi}{2}, \lambda= \pm \pi\right\}$ in the $x_{1} x_{3}-$ plane. The chart $\left(\Omega_{\iota}, \varphi_{\iota}\right)$ covers the sphere except for the set $\Gamma_{\iota}$, and the chart $\left(\Omega_{\kappa}, \varphi_{k}\right)$ similarly covers the sphere with the exception of a set $\Gamma_{\kappa}$. Hence the two charts $\left\{\left(\Omega_{l}, \varphi_{l}\right)\right.$, $\left.\left(\Omega_{\kappa}, \varphi_{\kappa}\right)\right\}$ together cover $S^{2}$ and they constitute an atlas. 


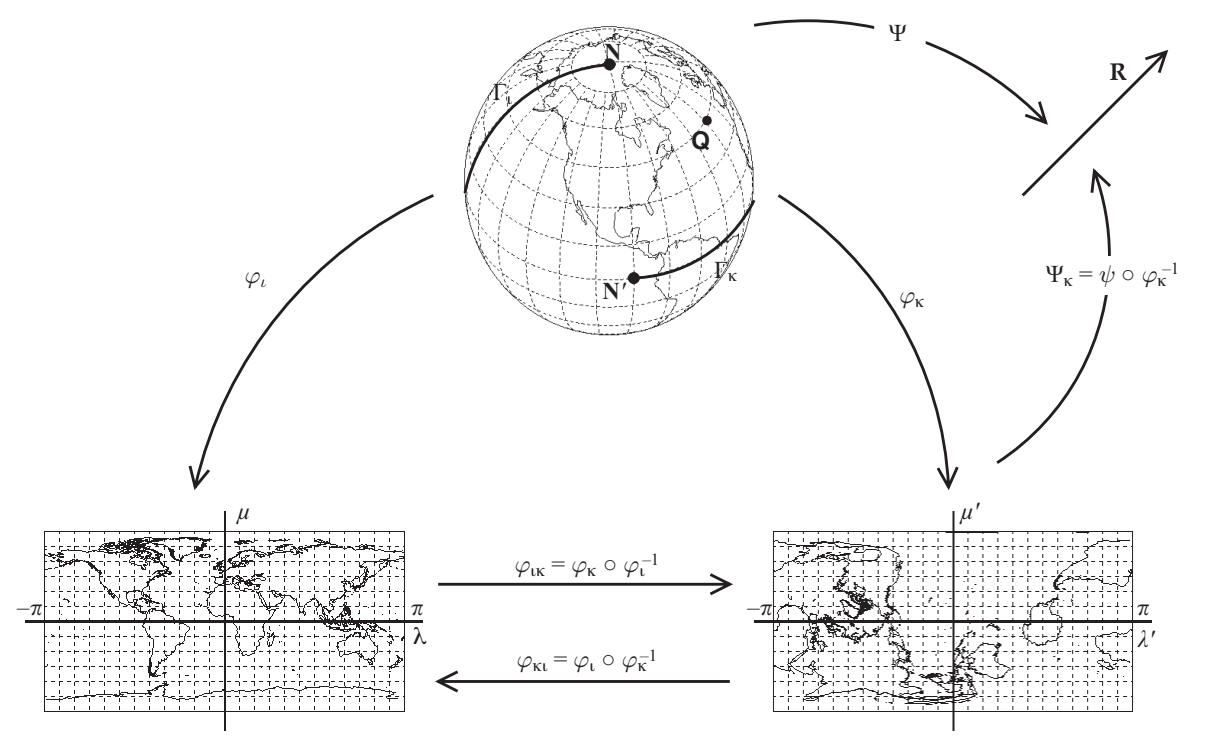

Fig. 1 . The set $\left\{\left(\Omega_{\iota}, \varphi_{\iota}\right),\left(\Omega_{\kappa}, \varphi_{k}\right)\right\}$ forms an atlas for $S^{2}: \Omega_{\iota} \cup \Omega_{\kappa}=S^{2}$.

The local coordinates associated with the chart $\left(\Omega_{\iota}, \varphi_{\iota}\right)$ are functions $\varphi_{l}: \Omega_{l}, \rightarrow R^{2}$, such that for $p \in S^{2}, \varphi_{\iota}(p)=\left(\varphi_{\iota, 1}(p), \varphi_{\iota, 2}(p)\right)=\left(x_{\iota}^{1}(p), x_{\iota}^{2}(p)\right)$ $=(\lambda(p), \mu(p))$ and $\varphi_{k}(p)=\left(x_{\kappa}^{1}(p), x_{\kappa}^{2}(p)\right)=\left(\lambda^{\prime}(p)\right.$; $\left.\mu^{\prime}(p)\right)$ are local coordinates with respect to the chart $\left(\Omega_{\kappa}, \varphi_{\kappa}\right)$ (Fig. 1).

To construct the map $\varphi_{\ell}: S^{2} \rightarrow U \subset R_{\ell}^{2}, \ell=\imath, \kappa$, a bijection with inverse $\varphi_{l}^{-1}: U_{\iota} \rightarrow S^{2}$ defined as $\varphi_{\iota}^{-1}\left(x_{\iota}^{1}, x_{\iota}^{2}\right)=\left(\sqrt{1-\left(x_{\iota}^{2}\right)^{2}} \cos x_{\iota}^{1}, \sqrt{1-\left(x_{\iota}^{2}\right)^{2}}\right.$ $\left.\sin x_{\iota}^{1}, x_{\iota}^{2}\right)$, and the $\varphi_{\kappa}^{-1}\left(x_{\kappa}^{1}, x_{\kappa}^{2}\right)=\left(\sqrt{1-\left(x_{\kappa}^{2}\right)^{2}} \cos x_{\kappa}^{1}\right.$ $\left.x_{\kappa}^{2}, \sqrt{1-\left(x_{\kappa}^{2}\right)^{2}} \sin x_{\kappa}^{1}\right)$, it is seen that every $\mathrm{U}_{\ell}$ is open. Hence each $\Omega_{\ell}$, is an open subset of $S^{2}$ and $\Omega_{\iota} \cup \Omega_{\kappa}$ cover $S^{2}$.

Given two charts of the atlas $\left\{\left(\Omega_{l}, \varphi_{\iota}\right),\left(\Omega_{\kappa}, \varphi_{k}\right)\right\}$ with $\left(\Omega_{\kappa} \cap \Omega_{\iota}\right) \neq \emptyset$, the transition maps $\varphi_{l \kappa}=\varphi_{\kappa} \circ \varphi_{\iota}^{-1}$ : $U_{\iota \kappa} \rightarrow U_{\kappa \iota}$ outline open sets of $R^{2}$ into $R^{2}$, where $U_{\iota \kappa}=$ $\varphi_{\iota}\left(\Omega_{\iota} \cap \Omega_{\kappa}\right)$ and $U_{\kappa l}=\varphi_{\kappa}\left(\Omega_{\kappa} \cap \Omega_{\iota}\right)$. This determines a differentiable structure for $S^{2}$, and $\varphi_{\iota \kappa}=\varphi_{\kappa} \circ \varphi_{\iota}^{-1}$ : is a diffeomorphism. It is then said that the atlas is of class $C^{k}$ if the transition functions are of $C^{k}$.

Let $x$ be any point of $U_{\iota k}$, and $\left(\varphi_{\iota \kappa}^{1}(x), \varphi_{\iota k}^{2}(x)\right)$ the coordinate of $\varphi_{\iota k}(x)$; then $\varphi_{l k}^{i}(x)$ is a continuous function on two variables. Now, if $p \in \Omega_{\iota} \cap \Omega_{\kappa}$ such that $x=\varphi_{\iota}(p)$, and since $\varphi_{\iota}(p) \in U_{\iota k}$, we have the relations

$$
\begin{gathered}
\varphi_{\iota \kappa}^{1}\left(x_{\iota}^{1}, x_{\iota}^{2}\right)=x_{\kappa}^{1}\left(x_{\iota}^{1}, x_{\iota}^{2}\right)=\lambda^{\prime}(\lambda, \mu)= \\
\tan ^{-1}\left[\frac{\sin \left(\lambda-\lambda_{\mathrm{o}}\right)}{\mu_{\mathrm{o}} \cos \left(\lambda-\lambda_{\mathrm{o}}\right)-\mu \sqrt{\frac{1-\mu_{\mathrm{o}}^{2}}{1-\mu^{2}}}}\right]
\end{gathered}
$$

$$
\begin{gathered}
\varphi_{\iota \kappa}^{2}\left(x_{\iota}^{1}, x_{\iota}^{2}\right)=x_{\kappa}^{2}\left(x_{\iota}^{1}, x_{\iota}^{2}\right)=\mu^{\prime}(\lambda, \mu)= \\
{\left[\mu \mu_{\mathrm{o}}+\sqrt{1-\mu^{2}} \sqrt{1-\mu_{\mathrm{o}}^{2}} \cos \left(\lambda-\lambda_{\mathrm{o}}\right)\right]}
\end{gathered}
$$

This is the transformation formula betwen the two local coordinate systems $\left(x_{\iota}^{1}, x_{\iota}^{2}\right)$ and $\left(x_{\kappa}^{1}, x_{\kappa}^{2}\right)$ defined on $\Omega_{\kappa} \cap \Omega_{\iota}$. To obtain the relations between the unprimed and primed coordinate of any point $Q$ on the sphere, Verkley (1984) examined the spherical triangle $N Q N^{\prime}$ and the application of the cosine rules to this triangle, deriving explicit expressions for the transformation between the two coordinate systems as given by (4) and (5).

Let $\Omega_{\iota} \cap \Omega_{\kappa} \neq \emptyset$, and set $J \varphi_{\iota \kappa}$ as the Jacobian matrix of map $\varphi_{\iota \kappa}$, so we can verify that

$\operatorname{det} J \varphi_{\iota \kappa}=\left|\begin{array}{ll}\frac{\partial \varphi_{\iota \kappa}^{1}}{\partial x_{\iota}^{1}} & \frac{\partial \varphi_{\iota \kappa}^{1}}{\partial x_{\iota}^{2}} \\ \frac{\partial \varphi_{\iota \kappa}^{2}}{\partial x_{\iota}^{1}} & \frac{\partial \varphi_{\iota \kappa}^{2}}{\partial x_{\iota}^{2}}\end{array}\right|=1$

Then $\operatorname{det} J \varphi_{\iota k}>0$. Hence, it is said that if manifold $S^{2}$ is oriented for every pair $\Omega_{\iota}, \Omega_{\kappa}$ of intersecting local coordinate neighbourhoods, det $J \varphi_{\iota \kappa}>0$.

Indeed we can regard the coordinate as a device to decide which of many functions $\psi$ on $S^{2}$ are to be differentiable. Since $\Omega_{\iota}$ is just a set, it makes no sense to ask that $\psi: \Omega_{\iota} \rightarrow R$ be differentiable (Matsushima, 1972; Loomis and Sterberg, 1990). However, we can consider the map $\Psi_{\iota}=\psi \circ \varphi_{\iota}^{-1}: \varphi_{\iota}\left(\Omega_{\iota}\right) \rightarrow R$. Then 
$\psi \circ \varphi_{\iota}^{-1}$ is a function defined on an open $\varphi_{\iota}\left(\Omega_{\iota}\right) \subset$ $R^{2}$, and we know what it means for such a function to be differentiable or smooth (see Fig. 1). Consider now what happens when we change coordinates to some other chart, lets say $\left(\Omega_{\kappa}, \varphi_{\kappa}\right)$ for convenience, assuming that $\Omega_{\iota}=\Omega_{\kappa}$. Then it is possible for $\psi \circ \varphi_{\iota}^{-1}$ to be differentiable but $\psi \circ \varphi_{k}^{-1}$ is not. To compare both, let $\psi \circ \varphi_{l}^{-1}=\psi \circ \varphi_{\kappa}^{-1} \circ\left(\varphi_{\kappa} \circ \varphi_{l}^{-1}\right)$ where the map $\varphi_{\kappa} \circ \varphi_{l}^{-1}: \varphi_{l}\left(\Omega_{l}\right) \rightarrow \varphi_{\kappa}\left(\Omega_{\kappa}\right)$ is a bijection between open subsets of $R^{2}$. Then a sufficient condition for $\psi \circ \varphi_{\iota}^{-1}$ to be differentiable if $\psi \circ \varphi_{\kappa}^{-1}$ is, is that $\varphi_{\kappa} \circ \varphi_{\iota}^{-1}$ is also differentiable. We often write $\Psi$ for the composite function $\psi \circ \varphi_{l}^{-1}$.

Lets take a curve $\tau:(-1,1) \rightarrow S^{2}$ with $\tau(0)=p$. In a local chart $\tau$ is given by $x_{\iota}^{i}=\tau^{i}(t)$. On the manifold $S^{2}$, one can define a vector $\mathbf{U}$ tangent to the parametrized curve $\tau$ at any point $p$ on the curve. The tangent vector $\mathbf{U}$ is given by a column vector $\mathbf{u}$ whose components $u_{\iota}^{i}$ are $\frac{d \tau^{i}}{d t}(0)$, $(i=1,2)$, with the initial condition $\tau(0)=p$. If we use another coordinate system corresponding to the chart $\left(\Omega_{\kappa}, \varphi_{\kappa}\right)$ by $\left\{x_{k}^{i}\right\}$ then the tangent vector $\mathbf{U}$ is given by a column vector $\mathbf{v}$ with components $v_{\kappa}^{i}$ According to the chain rule, the column vectors $\mathbf{u}$ and $\mathbf{v}$ are related by $v_{\kappa}^{i}=u_{\iota}^{j} \frac{\partial x_{\kappa}^{i}}{\partial x^{j}}$. The expression $u_{\iota}^{j} \frac{\partial}{\partial x_{\iota}^{j}}$ is the partial differential operator in the direction of the tangent vector.

The space $T_{p} S^{2}$ is called the tangent space of $S^{2}$ at $p$, and $T_{p} S^{2}$ is a two-dimensional vector space. For each $\mathbf{u} \in T_{p} S^{2}$ we shall write $\mathbf{u}=u_{\iota}^{i} \frac{\partial}{\partial x_{\iota}^{j}}=u^{1} \frac{\partial}{\partial \lambda}+$ $u^{2} \frac{\partial}{\partial \theta}$, where $u_{\iota}^{i}$ are the contravariant components of u. It is well known that on the manifold $S^{2}$ an inner product is defined at each tangent space $T_{p} S^{2}$. Now lets present a basis in which we denote the coordinate system corresponding to the chart $\left(\Omega_{\iota}, \varphi_{\iota}\right)$ by $\left\{x_{\iota}^{i}\right\}=$ $(\lambda, \mu)$, and for any $\psi: S^{2} \rightarrow R$ define the vectors $\left(\frac{\partial}{\partial x_{i}^{j}}\right)_{p}$ by $\left(\frac{\partial}{\partial x_{\iota}^{j}}\right)_{p} \psi=\left[\left(\frac{\partial \psi \circ \varphi_{\iota}^{-1}}{\partial x_{\iota}^{i}}\right)\right]_{\varphi_{\iota}(p)}$, so that they are independent since $\left(\frac{\partial}{\partial x_{\iota}^{j}}\right)_{p} x_{\iota}^{j}=\delta_{i}^{j}$. Let $\hat{n}_{\iota}=\left(\sqrt{1-\left(x_{\iota}^{2}\right)^{2}} \cos x_{\iota}^{1}\right.$, $\left.\sqrt{1-\left(x_{\iota}^{2}\right)^{2}} \sin x_{\iota}^{1}, x_{\iota}^{2}\right)$ be the outward normal to $S^{2}$ in $R^{3}$; without any loss of generality we may assume that the vectors $\left\{e_{\lambda}=\frac{\partial \hat{n}_{l}}{\partial x_{\iota}^{1}}, e_{\mu}=\frac{\partial \hat{n}_{\iota}}{\partial x_{\iota}^{2}}\right\}$ form a basis for $T_{p} S^{2}$.

We will denote the vector space of a vector field on $S^{2}$ by $\Gamma\left(S^{2}\right)$. A tangent vector field on $S^{2}$ is a smooth map u: $S^{2} \rightarrow T S^{2}$ such that, for any $x \in S^{2}, \mathbf{u}(x)$ $\in T_{x} S^{2}$. At the chart $\left(\Omega_{\iota}, \varphi_{\iota}\right)$, for $x \in \Omega_{\iota}$ the vector functions $\mathbf{u} \in T_{x} S^{2}$ and $\mathbf{v} \in \Gamma\left(S^{2}\right)$ have components $\mathbf{u}=u_{p}^{1} e_{\lambda}+u_{\mathrm{p}}^{2} e_{\mu}$ and $\mathbf{v}=v_{p}^{1} e_{\lambda}+v_{p}^{2} e_{\mu}$, respectively, being these $u_{p}^{i}=\mathbf{u}_{p}\left(x_{\imath}^{i}\right)=\left(u_{\lambda}, u_{\mu}\right)$ the components of $\mathbf{u}_{p}$ as the vectors of the unitary base indicated by $e_{\lambda}$, and $e_{\mu}$ in the directions $\lambda$ and $\mu$, respectively.

Let us recall that an oriented Riemannian manifold is a pair $\left(S^{2}, g\right)$ where $S^{2}$ is the oriented compact manifold and $g$ a Riemannian metric on $S^{2}$, which assigns a length $|\mathbf{v}|_{g(p)} \in R^{+}$. The $g$ on $S^{2}$ is a smooth $(2,0)$-tensor field on $S^{2}$ such that for any $p \in S^{2}$, $g_{p}: T_{p}\left(S^{2}\right) \times T_{p}\left(S^{2}\right) \rightarrow R$ is a scalar product on the tangent space $T_{p}\left(S^{2}\right)$, and in any chart $\left(\Omega_{\iota}, \varphi_{\iota}\right)$ of $S^{2}$, its components

$g_{i j}=\left(\begin{array}{cc}1-\mu^{2} & 0 \\ 0 & 1\end{array}\right)$

form a symmetric matrix, with its inverse denoted by $\left(g^{i j}\right)=\left(g_{i j}\right)^{-1}$, and $g=\operatorname{det}\left(g_{i j}\right)=1-\mu^{2}$. The length of a tangent vector $\mathbf{v} \in T_{p} S^{2}$ is defined as usual, $|\mathbf{v}|=g_{p}$ $(\mathbf{v}, \mathbf{v})^{1 / 2}=(\mathbf{v} \cdot \mathbf{v})^{1 / 2}$. Moreover, the inner product on $T$ $S^{2}$ is given by $\mathbf{u} \cdot \mathbf{v}=g_{i j} u^{i} v^{j}$, for $\mathbf{u}, \mathbf{v} \in T S^{2}$.

Let $\left(S^{2}, g\right)$ be the smooth Riemannian manifolds of $S^{2}$. Let us now recall some operators arising in partial differential equations on the sphere as manifold. Given the scalar function $\psi: S^{2} \rightarrow R$, the gradient of $\psi$, is given by the vector field grad $\psi$ : $S^{2} \rightarrow T_{p} S^{2}$, for which

$$
\begin{aligned}
\operatorname{grad} \psi= & \sum_{k, l}^{2}\left(g^{k l} \frac{\partial \Psi}{\partial x_{\iota}^{l}}\right) \frac{\partial}{\partial x_{\iota}^{l}}=\frac{1}{\sqrt{1-\mu^{2}}} \frac{\partial \Psi}{\partial \lambda} \hat{e}_{\lambda} \\
& +\sqrt{1-\mu^{2}} \frac{\partial \Psi}{\partial \mu} \hat{e}_{\mu}
\end{aligned}
$$

where $\hat{e}_{\lambda}=\frac{1}{\sqrt{1-\mu^{2}}} \mathrm{e}_{\lambda}$ and $\hat{e}_{\mu}=\sqrt{1-\mu^{2}} e_{\mu}$.

If $\mathbf{u} \in \Gamma\left(S^{2}\right)$, the divergence of $\mathbf{u}$ is the function on $S^{2}$ which on the chart $\left(\Omega, \varphi_{\iota}\right)$ is given by

$\operatorname{div} \mathbf{u}=\frac{1}{\sqrt{g}} \sum_{i} \frac{\partial}{\partial x_{\iota}^{i}}\left(u^{i} \sqrt{g}\right)=\frac{1}{\sqrt{1-\mu^{2}}} \frac{\partial u_{\lambda}}{\partial \lambda}+\frac{\partial u_{\mu} \sqrt{1-\mu^{2}}}{\partial \mu}$

A linear connection $D$ on $S^{2}$ is a map $D: T\left(S^{2}\right) \times$ $\Gamma\left(S^{2}\right) \rightarrow T\left(S^{2}\right)$ called the covariant derivative and the usual notation for $D(U, V)$ is $D_{U} V$. Let $\left(\Omega, \varphi_{\imath}\right)$ be a chart and as one can observe, the vectors $\left(e_{\lambda}=\frac{\partial}{\partial \lambda}\right.$, $\left.e_{\mu}=\frac{\partial}{\partial \mu}\right)$ can be nonconstant. An easy notation is $\operatorname{set} \nabla_{i}$ $=\mathrm{D} \frac{\partial}{\partial x^{i}}($ e.g. Hebey, 2000). There are smooth functions $\Gamma_{i j}^{k}: \Omega_{\iota} \rightarrow R$ such that for any $i, j$, and any $p \in \Omega_{\iota}$,

$$
D_{\frac{\partial}{\partial x_{i}^{i}}}\left(\frac{\partial}{\partial x_{\iota}^{i}}\right)(p)=\Gamma_{i j}^{k}(p)\left(\frac{\partial}{\partial x_{\iota}^{k}}\right)_{p}
$$


where $\Gamma_{i j}^{k}$ are the Christoffel symbols, defined by $\Gamma_{i j}^{k}$ $=\frac{1}{2} \sum_{l=1}^{2} g^{k l}\left(\frac{\partial g_{l j}}{\partial x^{i}}+\frac{\partial g_{i l}}{\partial x_{2}^{j}}-\frac{\partial g_{i j}}{\partial x_{1}^{i}}\right)$. On the chart $\left(\Omega_{\nu}, \varphi_{\iota}\right)$, we have $\Gamma_{11}^{1}=0, \Gamma_{11}^{2}=-2 \mu \sqrt{1-\mu^{2}}, \Gamma_{12}^{1}=\cot \theta=\frac{\mu}{\sqrt{1-\mu^{2}}}$, $\Gamma_{12}^{2}=0, \Gamma_{22}^{1}=0$ and $\Gamma_{22}^{2}=0$

The fundamental operator which we study is the Laplacian $\triangle$, then for real or complex valued functions, $\triangle$ is the Laplace-Beltrami operator on $S^{2}$ and it is given by

$$
\begin{aligned}
\Delta_{g} \psi= & -g^{i j}\left(\frac{\partial^{2} \psi}{\partial x_{\iota}^{i} \partial x_{\iota}^{j}}-\Gamma_{i j}^{k} \frac{\partial \psi}{\partial x_{\iota}^{k}}\right)= \\
& \frac{1}{\left(1-\mu^{2}\right)} \frac{\partial^{2} \Psi}{\partial \lambda^{2}}+\frac{\partial}{\partial \mu}\left[\left(1-\mu^{2}\right) \frac{\partial \Psi}{\partial \mu}\right]
\end{aligned}
$$

This operator satisfies some properties: $\Delta_{g}$, is selfadjoint, symmetric and non-negative (Aubin, 1998). Thus, the operators div, grad and $\Delta_{g}$ on the manifold $S^{2}$ have the conventional meaning.

Let $\left(S^{2}, g\right)$ be a compact oriented Riemannian manifold, with metric $g$. The metric and the orientation are combined to give a volume element $d v_{g}$ on $S^{2}$, which can be used to integrate functions on $\left(S^{2}, g\right)$. In order to apply the integral calculus on the oriented manifold $S^{2}$, we define a volume element to be a twoform $\omega=d v$ which is defined on all of $S^{2}$. For every $\operatorname{chart}\left(\Omega_{\ell}, \varphi_{\ell}\right)$ which is consistently oriented with $S^{2}$, the coordinate expresion for $\omega=d v_{l}$ is $\Phi_{l} d x_{l}^{1} \Lambda d x_{l}^{2}$ where $\Phi_{l}$ is a partition of unity subordinate to the covering $\left\{\Omega_{l}\right\}, l=\iota, \kappa$.

On the Riemannian manifold $\left(S^{2}, g\right)$, at the chart $\left(\Omega_{l}, \varphi_{l}\right)$; a volume form $\eta=d v_{g}$ defines a Lebesgue measure on $S^{2}$ by $d v_{g}=\eta=\sqrt{g} d x_{l}^{1} \Lambda d x_{l}^{2}$. Then

$\int_{S^{2}} \psi d v_{g}=\sum_{l} \int_{\varphi_{l}\left(\Omega_{l}\right)}\left(\Phi_{l} \sqrt{g} \psi\right) \circ \varphi_{l}^{-1} d x_{l}$

where $d x_{l}=d x_{l}^{1} d x_{l}^{2}$ defines a Lebesgue measure on $R^{2}$.

Let $\mathrm{C}^{\infty}\left(S^{2}\right)$ denote the set of infinitely differentiable functions of compact support $\psi(x)$. At $\mu= \pm 1$ the functions are smooth, together with the periodic boundary condition at $\lambda$ with period $2 \pi$. If we define the usual Hilbert space $L^{2}\left(S^{2}\right)$ to be the completion of $\mathrm{C}^{\infty}\left(S^{2}\right)$ with respect to the inner product

$<f, g>=\int_{S^{2}} f g^{*} d v_{g}$,

and norm $\|f\|=\left\{\int_{s^{2}}|f|^{2} d v_{g}\right\}^{1 / 2}, g^{*}$ is the complex conjugate of function $g$. Let $\left(S^{2}, g\right)$ be the compact Riemannian manifold and $d v_{g}$ the Riemannian volume element. Then functional spaces (Sobolev and the Holder spaces) can be defined on $S^{2}$ as well
(Skiba, 2012). For each $p \in \mathbf{R}$ with $1 \leq p<\infty$ we associate a Banach space

$$
\begin{aligned}
L^{p}\left(S^{2}\right)= & \left\{\psi: S^{2} \rightarrow \mathbf{R} ; \psi\right. \text { measurable and } \\
& \left.\int_{S^{2}}|f|^{p}<\infty\right\},
\end{aligned}
$$

with respect to the norm

$$
\|f\|_{p}=\left[\int_{S^{2}}|f|^{p} d v_{g}\right]^{\frac{1}{p}}
$$

and ess sup $|\mathrm{f}|<\infty$ if $p=\infty . L^{2}\left(T S^{2}\right)$ represent the Hilbert space of the vector fields $U: S^{2} \rightarrow T S^{2}$ endowed with the inner product in $L^{2}\left(S^{2}\right)$ induced by $g$ in $T_{p}\left(S^{2}\right)$ (see Díaz and Tello, 1999; Hebey, 2000).

We now turn to the eigenvalue problems for $\Delta_{g}$ : We usually seek to find all eigenvalues $\gamma$ for which there is an eigenfunction $Y$ such that $\Delta_{g} Y=-\gamma \mathrm{Y}$. Then, which information about geometry of $\left(S^{2}, g\right)$ is encoded by the eigenvalues?. The structure of eigenfunctions: $L^{p}$ norms and relations to $\mathrm{RH}$ waves or modons.

Global harmonic analysis is the study of the spectral theory of the Laplacian $\Delta_{g}$ on a compact Riemannian manifold $\left(S^{2}, g\right)$, and its relation to the global geometric structure. Since $\left(S^{2}, g\right)$ is compact, there exists an orthonormal basis $\left\{Y_{j}\right\}$ of smooth eigenfunctions and the spectrum of $\Delta_{g}$ is a discrete set $\left\{\gamma_{0}=0<\gamma_{1} \leq \gamma_{2} \leq \gamma_{3} \leq \ldots\right\}$. Recent developments show that the non-zero eigenvalues also contain substantial geometric and analytic information. The solution modon constructed by Tribbia (1984), Verkley $(1984,1987,1990)$ and Neven (1993) proposed the use of eigenfunctions $\left\{Y_{j}\right\}$ as basic geometric structures.

The space of spherical harmonics of degree $n$ on $S^{2}$, which coincides with the eigenspace of operator $\Delta_{g}$ corresponding to the eigenvalue $\gamma_{\mathrm{n}}=\chi_{\mathrm{n}}=n(n+1)$, is denoted by $\mathbf{H}_{\mathbf{n}}$. Self-adjoint operators have the property that its eigenfunctions with different eigenvalues are orthogonal, which implies that the eigenspaces $\mathbf{H}_{\mathbf{n}}$ are orthogonal and have $2 n+1$ dimensions. On the sphere, the homogeneous harmonic polynomials span the set of all polynomials, which in turn are dense in $L^{2}$. Our spherical harmonics therefore span $L^{2}$. If we take a basis within each eigenspace then this collection will give a basis for $L^{2}$ of the sphere. The harmonics spherical term was introduced by Kelvin on potentials studies (Hobson, 1931) and is 
understood as the development of a function in terms of this series of spherical harmonics.

The spaces $\mathbf{H}_{\mathbf{n}}$ and $\mathbf{H}_{\mathbf{k}}(n \neq k)$ are mutually orthogonal in $L^{2}\left(S^{2}\right)$. Then there is the orthogonal projection $Y_{n}: L^{2}\left(S^{2}\right) \rightarrow \mathbf{H}_{\mathrm{n}}$, and so smooth functions $\Psi \in L^{2}\left(S^{2}\right)$ on the sphere $S^{2}$ have a development in spherical harmonics,

$\Psi(\lambda, \mu)=\sum_{n=1}^{\infty} Y_{n}(\lambda, \mu)$

where $Y_{n}(\lambda, \mu)=\sum_{m=-n}^{n} \Psi_{n}^{m} Y_{n}^{m}(\lambda, \mu)$ is the homogeneous spherical polynomial of degree $n$ from $\mathbf{H}_{\mathbf{n}}$, and $\left.\Psi_{n}^{m}=<\Psi, Y_{n}^{m}\right\rangle$ is the Fourier coefficient of $\Psi$. The $2 n+1$ spherical harmonics

$Y_{n}^{m}(\lambda, \mu)=C_{n m} P_{n}^{m}(\mu) e^{i m \lambda}$

of degree $n$ and zonal number $m(-n \leq m \leq n)$ form an orthonormal basis in $\mathbf{H}_{\mathbf{n}}$. Here the numbers $C_{n m}$ are the normalizers in $L^{2}\left(S^{2}\right)$, given by $C_{n m}=$ $\left(\frac{2 n+1}{4 \pi} \frac{(n-m) !}{(n+m) !}\right)^{\frac{1}{2}}$, and $P_{\mathrm{n}}^{m}$ are the associated Legendre functions given by

$P_{n}^{m}(\mu)=\frac{\left(1-\mu^{2}\right)^{\frac{m}{2}}}{2^{n} n !} \frac{d^{n+m}}{d \mu^{n+m}}\left(\mu^{2}-1\right)^{n}$

Considering that an oriented compact Riemannian manifold is a pair $\left(S^{2}, g\right)$ where $S^{2}$ is the oriented compact manifold and $g$ a Riemannian metric on $S^{2}$, we can define in it covariant derivatives and various notions of curvature. When a manifold also has a group structure (so that multiplication and inversion are smooth), a very interesting structure called a Lie group (Bihlo, 2007; Bihlo and Popoych, 2012) arises. Even if a manifold $S^{2}$ is not a Lie group, there may be an action $:: \mathrm{G} \times S^{2} \rightarrow S^{2}$ of a Lie group $\mathrm{G}$ on $S^{2}$, and under certain conditions $S^{2}$ can be viewed as a "quotient" $\mathrm{G} / \mathrm{K}$, where $\mathrm{K}$ is a subgroup of G (Richtmyer, 1981). When $S^{2} \cong \mathrm{G} / \mathrm{K}$ as above, certain notions on $\mathrm{G}$ can be transported to $S^{2}$, then we say that $S^{2}$ is a homogeneous space. As an example of the last point we could mention the theory of spherical harmonic expansion on the $S^{2}$, which is a homogeneous space for the rotation group $\mathrm{O}(\mathrm{n}+1)$. The surface spherical harmonics are eigenfunctions for the Laplace-Beltrami operator, which is a rotation invariant (Helgason, 1984).
Harmonic analysis is concerned with the representation of functions as the superposition of basic waves, the study and generalization of the notions of Fourier series as well as the Fourier transforms.

Elements of harmonic analysis on the sphere can be found at Stein and Weiss (1971). After introducing the manifold $S^{2}$ and the Riemannian manifolds $\left(S^{2}, g\right)$, a general type of spaces (Besov and Triebel-Lizorkin spaces) on the sphere may also be introduced (Narcowich et al., 2006). Using the power of a Laplace operator, the Sobolev space on Riemannian manifolds can also be incorporated as a field currently undergoing great development (Aubin, 1998; Hebey, 2000).

\section{Exact solutions to the barotropic vorticity equa- tion on the manifold $\boldsymbol{S}^{2}$}

Let $\left\{\left(\Omega_{b}, \varphi_{l}\right)\right\}, \ell=\iota, \kappa$ be an atlas of $S^{2}$ and $\psi: S^{2} \rightarrow R$ the streamfunction of class $C^{r}$. We can consider that the map $\Psi=\psi \circ \varphi_{\iota}^{-1}: \varphi_{\iota}\left(\Omega_{\iota}\right) \rightarrow R$ and $\psi \circ \varphi_{\iota}^{-1}$ is the streamfunction defined on an open $U_{\iota}=\varphi_{\iota}\left(\Omega_{\iota}\right) \subset R^{2}$ and that it is of class $C^{r}$.

To simulate the time evolution of a two-dimensional nondivergent and inviscid flow for a rotating sphere, $S^{2}$ is governed by a non-linear barotropic vorticity equation, which can be written in the non-dimensional form as

$\frac{\partial \Delta \Psi}{\partial \mathrm{t}}+J(\Psi, \Delta \Psi+2 \mu)=0$

where $J(c, q)=\frac{\partial c}{\partial \lambda} \frac{\partial q}{\partial \mu}-\frac{\partial c}{\partial \mu} \frac{\partial q}{\partial \lambda}=(\mathrm{k} \times \nabla c) \cdot \nabla q=\mathbf{u} \cdot \nabla q$ is the jacobian, $\mathbf{u}=\mathrm{k} \times \nabla c=\left\{u_{\lambda}, u_{\mu}\right\}=\left\{-\sqrt{1-\mu^{2}} \frac{\partial c}{\partial \mu}\right.$, $\left.\frac{1}{\sqrt{1-\mu^{2}}} \frac{\partial c}{\partial \lambda}\right\}$ is a tangent velocity vector, $\operatorname{grad} c=\nabla c=$ $\left\{\frac{1}{\sqrt{1-\mu^{2}}} \frac{\partial c}{\partial \lambda}, \sqrt{1-\mu^{2}} \frac{\partial c}{\partial \mu}\right\}, c=\Psi, \xi=\Delta \Psi=\operatorname{div} \operatorname{grad} \Psi$, is the relative vorticity, $q=\Delta \Psi+2 \mu$ is the absolute vorticity and $\mathbf{k}$ is a unit outward normal vector. The velocity vector field $\mathbf{u}$ having the components $\left(u_{\lambda}, u_{\mu}\right)$ is solenoidal: $\nabla \cdot \mathbf{u}=0$. Throughout decades the nonlinear barotropic vorticity equation has been successfully used to describe low frequencies and large-scale barotropic processes of atmospheric dynamics. Despite the simplicity to this nonlinear equation, it contains the principal elements that describe the complexity of atmospheric behavior (Simmons et al., 1983; Skiba, 1997). The four types of exact solutions to Eq. (1) known up to now are described below: 
- The zonal flows and Rossby-Haurwitz (RH) waves (Haurwitz, 1949), called classical solutions, diferentiated from the generalized solutions which are not so smooth.

- The first generalized solutions of Eq. (6), kown as modons, were originally constructed by Tribbia (1984) and Verkley $(1984,1987,1990)$ by using two eigenfunctions for the Laplace operator of different degrees.

- Later on, Neven (1992) gave generalized solutions in the form of a quadrupole modon.

- Wu and Verkley (1993) constructed generalized global solutions composed of two RH waves (Pérez-García and Skiba, 1999).

In the present work, zonal flows, homogeneous spherical polynomials flows, RH waves, and modons on the manifold $S^{2}$ are considered.

\subsection{Classical solutions}

Let us consider the zonal flows, homogeneous spherical polynomials flows and Rossby-Haurwitz (RH) waves.

Proposition (zonal flow). Let $\left\{\left(\Omega_{l}, \varphi_{l}\right)\right\}, \ell=\iota, \kappa$ be an atlas of $S^{2}$ and the streamfunction $\psi: S^{2} \rightarrow R$ of class $C^{r}$. Then the zonal flow map $\Psi_{\iota}=\psi \circ \varphi_{\iota}^{-1}$ : $U_{\iota} \subset R_{\iota}^{2} \rightarrow R$ of $C^{r}$ defined as

$\Psi_{\iota}(\lambda, \mu)=\sum_{\mathrm{n}=0}^{N} b_{n} P_{n}^{0}(\mu)$

is an exact solution of the vorticity Eq. (6) for any $b_{n}$.

Proof. The demonstration, obtained from Eq. (6), is quite trivial.

Proposition (homogeneous polynomials). Let $\left\{\left(\Omega_{\ell}, \varphi_{\ell}\right)\right\}, \ell=\iota, \kappa$ be an atlas of $S^{2}$ and the streamfunction $\psi: S^{2} \rightarrow R$ of class $C^{r}$. Then the homogeneous spherical polynomial map $\Psi_{\iota}=$ $\psi \circ \varphi_{\iota}^{-1}: U_{\iota} \subset R_{\iota}^{2} \rightarrow R$ of degree $\mathrm{n} \geq 2$ defined as

$\Psi_{\iota}(\lambda, \mu, t)=\sum_{m=n}^{N} a_{m} Y_{n}^{m}(\lambda-c t, \mu) \in \mathbf{H}_{n}$

is an exact solution to the vorticity Eq. (6), where $a_{m}$ can be a complex factor and

$\mathrm{c}=-\frac{2}{\chi_{\mathrm{n}}}$

is the angular phase speed.
Proof. Given $\Psi_{\iota} \in \mathbf{H}_{n}$, we define $\Psi_{\iota}(\lambda, \mu, t)=$ $\Psi_{n}(\lambda-c t, \mu)=\sum_{m=n}^{n} a_{m} Y_{n}^{m}(\lambda-c t, \mu)$, then $\frac{\partial \Psi_{n}}{\partial t}=-2 \Psi^{\prime}$, and $\frac{\partial \Psi_{n}}{\partial \lambda}=\Psi^{\prime}$, where $\Psi^{\prime}=\sum_{m=n}^{n} i m a_{m} Y_{n}^{m}(\lambda-c t, \mu)$. If, in addition, we have the following expression

$$
\begin{aligned}
\Delta \Psi_{\iota}= & -\chi_{n} \Psi_{n} ; \frac{\partial \Delta \Psi_{\iota}}{\partial \mu}=-\chi_{n} \frac{\partial \Psi_{n}}{\partial \mu} ; \Delta \Psi_{\iota}+2 \mu= \\
& -\chi_{n} \Psi_{\iota}+2 \mu ; \frac{\partial \Delta \Psi_{\iota}}{\partial t}=c \chi_{n} \Psi^{\prime}
\end{aligned}
$$

we have

$$
\begin{aligned}
\Delta \frac{\partial \Psi_{\iota}}{\partial t}= & \chi_{n} c \Psi^{\prime}=J\left(-\chi_{n} \Psi_{n}+2 \mu, \Psi_{n}\right)= \\
& -2 \frac{\partial \Psi_{n}}{\partial \lambda}=-2 \Psi^{\prime}
\end{aligned}
$$

from BVE (Eq. (6)). It follows that $\mathrm{c}=-\frac{2}{\chi_{\mathrm{n}}}$.

Proposition (Rossby-Haurwitz waves). Let $\left\{\left(\Omega_{l}, \varphi_{l}\right)\right\}, \ell=\iota, \kappa$ be an atlas of $S^{2}$ and the streamfunction $\psi: S^{2} \rightarrow R$ of class $C^{\infty}$. Then, the map $\Psi_{\iota}=$ $\psi \circ \varphi_{\iota}^{-1}: U_{\iota} \subset R_{\iota}^{2} \rightarrow R$ of $C^{\infty}$, defined as

$$
\begin{aligned}
\Psi_{\iota}(\lambda, \mu, t)= & -\omega \mu+\sum_{m=-n}^{\mathrm{n}} a_{m} Y_{n}^{m} \\
& (\lambda-c t, \mu) \in \mathbf{H}_{0} \oplus \mathbf{H}_{n}
\end{aligned}
$$

with $n \geq 1$; is called Rossby-Haurwitz (RH) waves. It is an exact solution of the vorticity Eq. (6) if the angular phase speed of the $\mathrm{RH}$ wave

$c=\omega-\frac{2(\omega+1)}{\chi_{n}}$.

Here $\omega$ is the super-rotation velocity and each $\mathbf{H}_{n}$ corresponds to the eigenvalue $\chi_{n}=n(n+1)$.

Proof. Here $\Psi_{\iota}$ is expressed by

$\Psi_{\iota}(\lambda, \mu, t)=-\omega \mu+\Psi_{n}(\lambda-c t, \mu)$

where $\Psi_{n}(\lambda-c t, \mu)=\sum_{m=n}^{n} a_{m} Y(\lambda-c t, \mu)$. We can notice that

$\Delta \Psi_{\iota}=2 \omega \mu-\chi_{n} \Psi_{n}=-\chi_{n} \Psi_{\iota}+\left(2 \omega-\chi_{n} \omega\right) \mu$

which implies that

$$
\begin{aligned}
\Delta \Psi_{\iota}+2 \mu= & -\chi_{n} \Psi_{\iota}-\chi_{n}\left(\omega-\frac{2(\omega+1)}{\chi_{n}}\right) \mu \\
& =-\chi_{n} \Psi_{\iota}+\left[\left(2-\chi_{n}\right) \omega+2\right] \mu .
\end{aligned}
$$


Furthermore:

$$
\begin{aligned}
\frac{\partial}{\partial \lambda} \Delta \Psi_{\iota}= & -\chi_{n} \frac{\partial \Psi_{\iota}}{\partial \lambda}, \Delta \frac{\partial \Psi_{\iota}}{\partial t}=\chi_{n} c \Psi^{\prime}, \frac{\partial}{\partial \mu} \Delta \Psi_{\iota} \\
& =\left(2-\chi_{n}\right) \omega-\chi_{n} \frac{\partial \Psi_{\iota}}{\partial \mu}
\end{aligned}
$$

where $\Psi^{\prime}=\sum_{m=n}^{n} \operatorname{ima}_{m} Y_{n}^{m}(\lambda-c t, \mu)$; so that from BVE (Eq. (6))

$$
\begin{gathered}
\Delta \frac{\partial \Psi_{\iota}}{\partial t}=-\mathrm{J}\left(\Psi_{\iota}, \Delta \Psi_{\iota}+2 \mu\right)=\frac{\partial \Psi_{\iota}}{\partial \mu} \frac{\partial \Delta \Psi_{\iota}}{\partial \lambda}- \\
\frac{\partial \Psi_{\iota}}{\partial \lambda} \frac{\partial \Delta \Psi_{\iota}}{\partial \mu}-2 \frac{\partial \Psi_{\iota}}{\partial \lambda}=\left[\frac{\partial \Psi_{\iota}}{\partial \mu}\left(-\chi_{n} \frac{\partial \Delta \Psi_{\iota}}{\partial \lambda}\right)-\right. \\
\left.\frac{\partial \Psi_{\iota}}{\partial \lambda}\left(\left(2-\chi_{n}\right) \omega-\chi_{n} \frac{\partial \Psi_{\iota}}{\partial \mu}\right)\right]-2 \frac{\partial \Psi_{\iota}}{\partial \lambda} .
\end{gathered}
$$

Hence

$$
\begin{aligned}
\chi_{n} c \Psi^{\prime} & =-\frac{\partial \Psi_{\iota}}{\partial \lambda}\left(2-\chi_{n}\right) \omega-2 \frac{\partial \Psi_{\iota}}{\partial \lambda}= \\
& -\left[\left(2-\chi_{n}\right) \omega+2\right] \Psi^{\prime}= \\
& -\left[-\omega \chi_{n}+2(\omega+1)\right] \Psi^{\prime}
\end{aligned}
$$

so that

$c=\omega-\frac{2(\omega+1)}{\chi_{n}}$

and thus the proposition is proved.

The streamfunction of the stationary $\mathrm{RH}(2,5)$ wave

$\Psi(\lambda, \mu)=-\omega \mu+a P_{n}^{m}(\mu) \cos (m \lambda)$

with the parameters defined by $(m, n)=(2,5)$, $a=.007$ and $\omega=\frac{2}{\sqrt{3}\left(\chi_{3}-2\right)}$ is given in Figure 2.

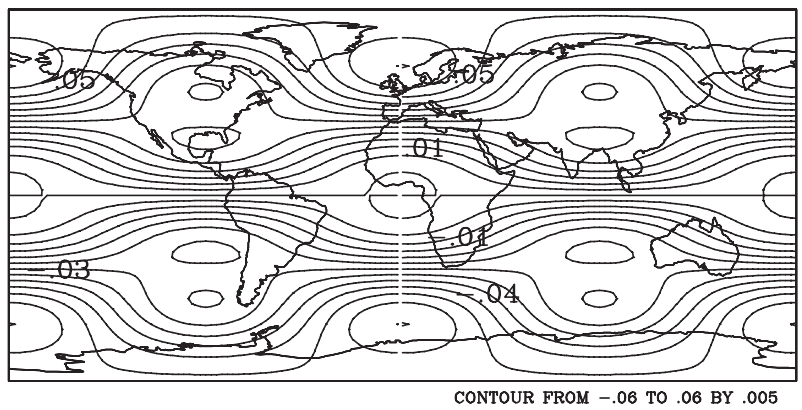

Fig. 2. Isolines of the streamfunction of Rossby-Haurwitz waves $(2,5)$.
Pérez and Skiba (2001) and Skiba and Pérez (2006) developed a numerical spectral method for the normal mode instability study of the arbitrary steady flow of an ideal nondivergent fluid on a rotating sphere, and Skiba and Pérez (2006) tested this method for the RH(2,5) wave. Pérez-García (2014) constructed a basic flow regarded as a sum of a zonally symmetric flow (Eq. 7) and a Rossby-Haurwitz wave component (Eq. 9).

\subsection{Generalized solutions}

Denote the spherical distance between two points of $S^{2}$ by $d(.,$.$) . Let N^{\prime}$ be the north pole of the chart coordinate $\left(\Omega_{v}, \varphi_{k}\right)$. Then a disk or inner region $S_{i}$ on the sphere is defined as $S_{i}=D\left(N^{\prime}, \phi_{a}\right)=\left\{\mathrm{s} \in S^{2} \mid\right.$ $\left.d\left(N^{\prime}, s\right)<\phi_{a}\right\}$, such that $0<\phi_{a} \leq \frac{\pi}{2}$. The solution modon is constructed (Tribbia, 1984; Verkley, 1984, 1987, 1990; Neven, 1993) to divide the sphere $S^{2}$ into two regions: an inner region $S_{i}$ centered around the pole $N^{\prime}$, and an outer region $S_{o}$ separated from the inner region by a boundary circle $\partial D\left(N^{\prime}, \phi_{a}\right)=\left\{\mathrm{s} \in S^{2} \mid\right.$ $\left.d\left(N^{\prime}, s\right)=\phi_{a}\right\}$, on which $\psi, q$ and $\psi^{\prime}$ are continuous.

For $S_{i}$, a solution of the Eq. (2) form is chosen with an eigenfunction $Y\left(\lambda^{\prime}, \mu^{\prime}\right)$, which has its singularity in the outer region. The same type of solution is chosen for the outer region, but such that $Y\left(\lambda^{\prime}\right.$, $\mu^{\prime}$ ) has its singularity in the inner region. Then both solutions are combined as smoothly as possible on the boundary circle $\partial D\left(N^{\prime}, \phi_{a}\right)$ (Tribbia, 1984; Verkley, 1984, 1987).

To construct the Verkley (1984) modon or the Neven (1992) cuadrupole modon on the manifold $S^{2}$, it is interpreted as:

Proposition. Let $\left\{\left(\Omega_{\ell}, \varphi_{\ell}\right)\right\}, \ell=\iota, \kappa$, be an atlas of $S^{2}$, and $\psi=\psi_{1}+\psi_{2}: S^{2} \rightarrow R$ the streamfunction of $C^{r}$. Then

$\Psi_{\iota}(\lambda, \mu)=\left(\psi_{1} \circ \varphi_{\kappa}^{-1}\right)\left(\lambda^{\prime}, \mu^{\prime}\right)+\left(\psi_{2} \circ \varphi_{\iota}^{-1}\right)(\lambda, \mu)$.

Proof. Let $\psi_{1}$ and $\psi_{2}$ be two real-value functions of class $C^{r}$ defined on the differential manifolds $S^{2}$. We define their sum by setting $\Psi_{\iota}=\left(\psi_{1}+\psi_{2}\right) \circ \varphi_{l}^{-1}$ $=\psi_{1} \circ \varphi_{l}^{-1}+\psi_{2} \circ \varphi_{l}^{-1}$ for any chart $\left(\Omega_{\iota}, \varphi_{\iota}\right)$. Since the sum of two functions of class $C^{r}$ on $S^{2}$ are functions of class $C^{r}$, the proof of this formula can be obtained by the expression

$\Psi_{\iota}(\lambda, \mu)=\left(\psi \circ \varphi_{l}^{-1}\right)(\lambda, \mu)=\psi_{1} \circ \varphi_{l}^{-1}(\lambda, \mu)+\psi_{2} \circ$ $\varphi_{l}^{-1}(\lambda, \mu)$ 


$$
\begin{aligned}
& =\left(\psi_{1} \circ \varphi_{\kappa}^{-1}\right) \circ\left(\varphi_{\kappa} \circ \varphi_{\iota}^{-1}\right)(\lambda, \mu)+\left(\psi_{2} \circ \varphi_{\iota}^{-1}\right)(\lambda, \mu) \\
& =\left(\psi_{1} \circ \varphi_{\kappa}^{-1}\right) \circ \varphi_{\iota \kappa}(\lambda, \mu)+\left(\psi_{2} \circ \varphi_{\iota}^{-1}\right)(\lambda, \mu) \\
& =\left(\psi_{1} \circ \varphi_{\kappa}^{-1}\right)\left(\lambda^{\prime}, \mu^{\prime}\right)+\left(\psi_{2} \circ \varphi_{\iota}^{-1}\right)(\lambda, \mu)
\end{aligned}
$$

where $\varphi_{\iota \kappa}(\lambda, \mu)=\left(\varphi_{\iota \kappa}^{1}(\lambda, \mu), \varphi_{\iota \kappa}^{2}(\lambda, \mu)\right)=\left(\lambda^{\prime}(\lambda, \mu)\right.$, $\left.\mu^{\prime}(\lambda, \mu)\right)$ •

Decompose now the streamfunctions into an eigenfunction part $\left(\psi_{1} \circ \varphi_{\kappa}^{-1}\right)\left(\lambda^{\prime}, \mu^{\prime}\right)=\mathrm{Y}_{\nu}\left(\lambda^{\prime}, \mu^{\prime}\right)$ and a zonal part $\left(\psi_{2} \circ \varphi_{\iota}^{-1}\right)(\lambda, \mu)=-\omega \mu+D$, where $-\omega \mu$ is a solid-body rotation and $D$ a constant. In chart $\left(\Omega_{\kappa}, \varphi_{\kappa}\right)$ with coordinates $\left(\lambda^{\prime}, \mu^{\prime}\right)$, the north pole $N^{\prime}$ moves along a circle of constant latitude with constant angular velocity $c_{\nu}$. In the primed coordinates, Verkley $(1984,1987)$ modons have the form

$\left(\psi_{1} \circ \varphi_{\kappa}^{-1}\right)\left(\lambda^{\prime}, \mu^{\prime}\right)=X\left(\lambda^{\prime}, \mu^{\prime}\right)=X^{d}\left(\mu^{\prime}\right) \cos \lambda^{\prime}+X^{m}\left(\mu^{\prime}\right)$ $\rightarrow \mathrm{Y}_{\nu}\left(\lambda^{\prime}, \mu^{\prime}\right)$

which consists of a dipole and a monopole component:

$$
\begin{aligned}
& X^{d}\left(\mu^{\prime}\right)=\left(\mathrm{c}_{\nu}-\omega\right) \sqrt{1-\mu_{\mathrm{a}}^{2}} \sqrt{1-\mu_{0}^{2}} f^{d}\left(\mu^{\prime}\right) \\
& X^{m}\left(\mu^{\prime}\right)=\left(\mathrm{c}_{\nu}-\omega\right) \mu_{0} \sqrt{1-\mu_{\mathrm{a}}^{2}} f^{m}\left(\mu^{\prime}\right)
\end{aligned}
$$

where $\mu_{0}=\operatorname{sen} \phi_{0}, \mu_{a}=\operatorname{sen} \phi_{a}$. The functions $f^{d}(\mu)$ and $f^{m}(\mu)$ are defined as

$$
f^{d}(\mu)=\left\{\begin{array}{c}
-b B(1,1, \mu)+(1+b)\left(\frac{1-\mu^{2}}{1-\mu_{\mathrm{a}}^{2}}\right)^{\frac{1}{2}}, \text { if } \mu \geq \mu_{a} \\
P(1,1,-\mu), \text { if } \mu<\mu_{a}
\end{array}\right.
$$

and

$$
f^{m}(\mu)=\left\{\begin{array}{c}
-b B(0,1, \mu)+(1+b) \frac{\mu^{-} \mu_{a}}{\sqrt{1-\mu_{\mathrm{a}}^{2}}} \\
-P\left(0,1,-\mu_{a}\right)+b B\left(0,1, \mu_{a}\right), \text { if } \mu \geq \mu_{a} \\
-P(1,1,-\mu), \text { if } \mu<\mu_{a}
\end{array}\right.
$$

where $b=\frac{\left(k^{2}+\frac{1}{4}\right)+2}{\alpha(\alpha+1)-2}$ and

$$
B(r, s, \mu)=\frac{P_{\alpha}^{r}(\mu)}{P_{\alpha}^{s}\left(\mu_{a}\right)} ; P(r, s, \mu)=\frac{P_{-0.5+i k}^{r}(\mu)}{P_{-0.5+i k}^{s}\left(-\mu_{a}\right)}
$$

The fact that
$\Psi(\lambda, \mu, t)=Y_{\nu}\left(\lambda^{\prime}, \mu^{\prime}\right)-\omega \mu+D$

is a solution to Eq. (6) is due to the work of Verkley (1984), which I will not reproduce in this paper. $Y_{\nu}$ is an eigenfunction of the Laplace-Beltrami operator and $\chi_{\nu}=-\nu(\nu+1)$ is the eigenvalue of $Y_{\nu}$. The Legendre functions $H(\mu)=P_{\nu}^{m}(\mu)$ and $H(\mu)=Q_{\nu}^{m}(\mu)$ are solutions to the Legendre differential equation of hypergeometric type, where $P_{\nu}^{m}(\mu)$ is a Legendre function of the first kind and $Q_{\nu}^{m}(\mu)$ is a Legendre function of the second kind for order $m$ such that $\nu$ is the complex degree. The explicit expresion for $P_{\nu}^{m}(\mu)$ and $Q_{\nu}^{m}(\mu)$ with $-1<\mu<1$ can be found in Abramowitz and Stegun (1965) or Verkley (1984).

By using a grid of $5 \times 5^{\circ}$ upon the local coordinate associated with the chart $\left(\Omega_{\kappa}, \varphi_{\kappa}\right)$, the Verkley 1984 modon was numerically generated. Using Eqs. (4) and (5) a workable Gaussian mesh of $(128,64)$ points upon the geographical coordinate group $\left(\Omega_{\iota}, \varphi_{\iota}\right)$ was also generated. This mesh was mapped onto the local coordinates system associated to the chart $\left(\Omega_{\kappa}, \varphi_{\kappa}\right)$. The values of $\Psi_{\kappa}$ were interpolated on the Gaussians points $(128,64)$ by implementing a ninepoint Lagrange interpolation scheme. The resulting function, i.e. the Verkley 1984 equatorial modon viewed on the geographical coordinate group $\left(\Omega_{\iota}, \varphi_{\iota}\right)$, is shown in Figure 3a. This small modon was defined by the following parameters:

$k=10, \alpha=10, \mu_{a}=\sin 66.14^{\circ}, \mu_{0}=0, \lambda_{0}=270^{\circ}$ and $D_{0}=0$.

A numerical spectral model was used to simulate this small-size modon in Pérez-García and Skiba (1999), and in Skiba and Pérez-García (2009) a numerical spectral method for normal mode stability study of ideal flows on a rotating sphere was tested for this isolated steady modon constructed by Verkley (1984).

Studies done by Illari (1984) and Crum and Stevens (1988) noted that the values of isentropic potential vorticity are relatively low and uniform in the blocking region. In our following argument we consider that Verkley's modon (1990) provides a better and more uniform description of atmospheric blocking. Our interest lays within the fields that build this phenomenon. These solutions are characterized by a region $S_{i}$ in which $q$ is constant, and an outer region $S_{o}$ separated from the inner region by the boundary 

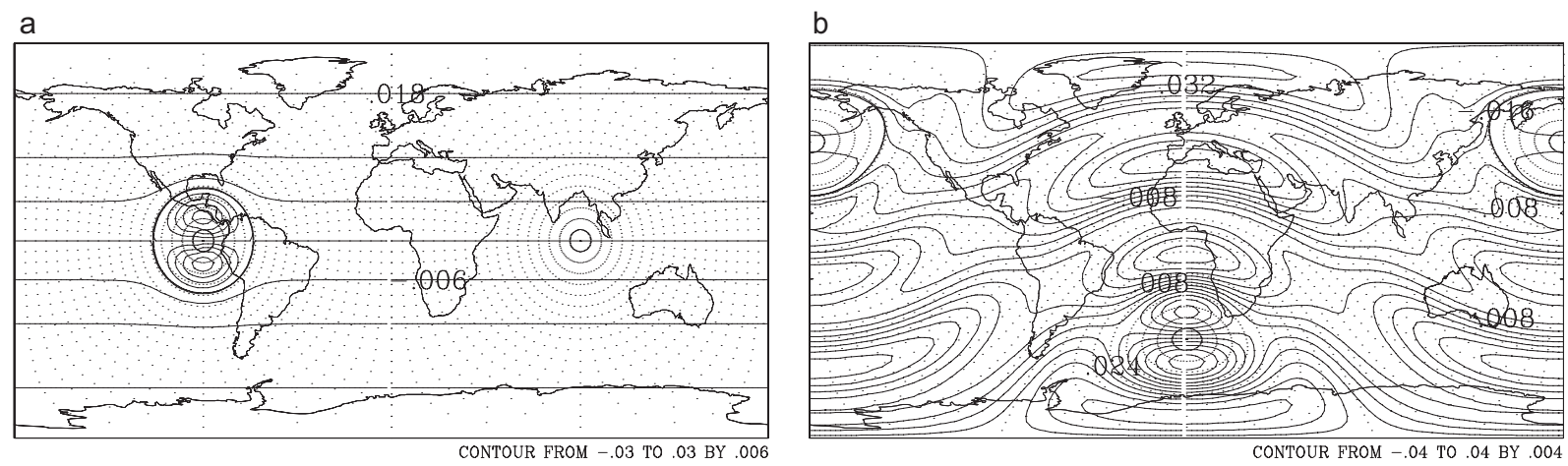

Fig. 3. Streamfunction isolines of equatorial Verkley modon (1984) with (a) $k=10$., $\alpha=10$., $\phi_{a}=66.14^{\circ}, \lambda_{0}=$ $270.0^{\circ}$ and $\phi_{0}=0 .^{\circ}$; the uniform Verkley modon (1990) at (b) $\sigma=8.06, \phi_{a}=\frac{5 \pi}{12}, \lambda_{0}=180.0^{\circ}$ and $\phi_{0}=45.0^{\circ}$. The curve points are the spherical coordinates relative to a rotated pole $N^{\prime}\left(270.0^{\circ}, 0 .^{\circ}\right)$ at (a) $N^{\prime}\left(180.0^{\circ}, 45 .^{\circ}\right)$ at (b) with respect to the original system.

circle $\partial D$, on which $\Psi$ and $q$ are both constant, i.e., $\Psi=d$ and $q=b$.

In the primed coordinates the Verkley (1990) modon has the form

$\Psi_{\kappa}\left(\lambda^{\prime}, \mu^{\prime}\right)=\left\{\begin{array}{c}Y_{o}\left(\lambda^{\prime}, \mu^{\prime}\right)-\omega_{o} \mu+D_{o} \text { at } S_{o} \\ Y_{i}\left(\lambda^{\prime}, \mu^{\prime}\right)-\omega_{i} \mu+D_{i} \text { at } S_{i}\end{array}\right.$

where solid-body rotation terms $\mu$ can be expressed in primed coordinates using Eqs. (4-5), such that in chart $\left(\Omega_{\iota}, \varphi_{\iota}\right)$ the eigenfuctions at the outer region and inner region are $\Delta^{\prime} Y_{o}=-\chi^{o} Y_{o} ; \Delta^{\prime} Y_{i}=e_{i}$, being $\chi^{o}$ and $e_{i}$ constants. Certain requirements of continuity must be met to generate these functions over the circle $\partial D$. We require continuity in $\phi^{\prime}$ and the first and second derivative in $\phi^{\prime}$ at $\phi_{a}$.

To construct the Verkley 1990 uniform modon on the manifold $S^{2}$, it is interpreted as:

Proposition. Consider an atlas $\left\{\left(\Omega_{\ell}, \varphi_{\ell}\right)\right\}, \ell=\iota$, $\kappa$, of $S^{2}$ and let $\psi=\psi_{1}+\psi_{2}: S^{2} \rightarrow \mathrm{R}$ be the streamfunction of $C^{r}$. Then

$\Psi_{\kappa}\left(\lambda^{\prime}, \mu^{\prime}\right)=\left(\psi_{1} \circ \varphi_{\kappa}^{-1}\right)\left(\lambda^{\prime}, \mu^{\prime}\right)+\left(\psi_{2} \circ \varphi_{\kappa}^{-1}\right)(\lambda, \mu)$

Proof. Let $\psi_{1}$ and $\psi_{2}$ be two real-value functions of class $C^{r}$ defined on the differential manifolds $S^{2}$. We define their sum by setting $\Psi_{\kappa}=\left(\psi_{1}+\psi_{2}\right) \circ \varphi_{\kappa}^{-1}=$ $\psi_{1} \circ \varphi_{\kappa}^{-1}+\psi_{2} \circ \varphi_{\kappa}^{-1}$ for any chart $\left(\Omega_{\kappa}, \varphi_{\kappa}\right)$. Since the sum of two functions of class $C^{r}$ on $S^{2}$ are functions of class $C^{r}$, the proof of the proposition follows from:

$\Psi_{\kappa}\left(\lambda^{\prime}, \mu^{\prime}\right)=\psi_{1} \circ \varphi_{\kappa}^{-1}\left(\lambda^{\prime}, \mu^{\prime}\right)+\psi_{2} \circ \varphi_{\kappa}^{-1}\left(\lambda^{\prime}, \mu^{\prime}\right)$

$$
\begin{aligned}
& =\left(\psi_{1} \circ \varphi_{\kappa}^{-1}\right)\left(\lambda^{\prime}, \mu^{\prime}\right)+\left(\psi_{2} \circ \varphi_{\iota}^{-1}\right) \circ\left(\varphi_{\iota} \circ \varphi_{\kappa}^{-1}\right)\left(\lambda^{\prime}, \mu^{\prime}\right) \\
& =\left(\psi_{1} \circ \varphi_{\kappa}^{-1}\right)\left(\lambda^{\prime}, \mu^{\prime}\right)+\left(\psi_{2} \circ \varphi_{\iota}^{-1}\right)(\lambda, \mu)
\end{aligned}
$$$$
\text { because }(\lambda, \mu)=\varphi_{\kappa \iota}\left(\lambda^{\prime}, \mu^{\prime}\right) \text { • }
$$

According to Verkley (1990), to express the modon in a more explicit manner, the functional forms $Y_{o}$ and $Y_{i}$ of the eigenfuntions are:

$$
\begin{aligned}
& Y_{o}\left(\lambda^{\prime}, \mu^{\prime}\right)=A_{0} S_{\sigma}^{1}\left(\theta^{\prime}\right) \cos \lambda^{\prime}+B_{0} S_{\sigma}^{0}\left(\theta^{\prime}\right) \\
& Y_{i}\left(\lambda^{\prime}, \mu^{\prime}\right)=A_{i} T^{1}\left(\theta^{\prime}\right) \cos \lambda^{\prime}+B_{i} T^{0}\left(\theta^{\prime}\right)
\end{aligned}
$$

where $A_{s}$ and $B_{s}$ are constant. The special functions $S_{\sigma}^{m}\left(\theta^{\prime}\right)=P_{\sigma}^{m}\left(-\cos \theta^{\prime}\right)$ and $T^{n}\left(\theta^{\prime}\right)$ were given by Verkley (1990).

We have also reproduced numerically the uniform modon constructed by Verkley (1990) using the parameters $\phi_{a}=\frac{5 \pi}{12}, \sigma=8.06 ; \omega_{o}=0.028$, and when the modon center is in the point $\lambda_{o}=180^{\circ}$, $\varphi_{o}=\frac{\pi}{4}($ Fig 3b).

\section{Conclusions}

The exact solutions of the barotropic vorticity equation on the rotating unit sphere as a compact differentiable manifold without boundary, which are zonal flows, homogeneous spherical polynomial flows, Rossby-Haurwitz waves and generalized solutions named modons, were represented in this paper. A concrete notion of local chart, change of charts, and atlas for the manifolds $S^{2}$ was developed. An atlas $\left\{\left(\Omega_{\iota}, \varphi_{\iota}\right),\left(\Omega_{\kappa}, \varphi_{\kappa}\right)\right.$. $\}$ was constructed for $S^{2}$, where every chart corresponds to a geographical coordinate 
group that covers most of $S^{2}$. The transition maps $\varphi_{\iota \kappa}=\varphi_{\kappa} \circ \varphi_{\iota}^{-1}: \varphi_{\iota}\left(\Omega_{\iota} \cap \Omega_{\kappa}\right) \rightarrow \varphi_{\kappa}\left(\Omega_{\iota} \cap \Omega_{\kappa}\right)$ and $\varphi_{\kappa \iota}=\varphi_{\iota} \circ \varphi_{\kappa}^{-1}: \varphi_{\kappa}\left(\Omega_{\kappa} \cap \Omega_{\iota}\right) \rightarrow \varphi_{\iota}\left(\Omega_{\kappa} \cap \Omega_{\iota}\right)$ were also constructed, and the exact solutions of the barotropic vorticity equation in a manifold context were studied. This work also formulates on the manifolds $S^{2}$ in terms of the stream function $\psi: S^{2} \rightarrow R$, for RH waves which are sufficiently smooth, and for Wu-Verkley waves and modons which are differentiably weak. $\mathrm{RH}$ waves as solutions $\psi \circ \varphi_{\iota}^{-1}$ of the barotropic vorticity equation on the manifolds $S^{2}$ are presented at the local coordinate associated with a chart $\left(\Omega_{\iota}, \varphi_{\iota}\right)$. The way in which the modon solution $\psi \circ \varphi_{\kappa}^{-1}$ can be constructed in the chart $\left(\Omega_{\kappa}, \varphi_{\kappa}\right)$, where $\psi$ is $C^{2}$, is investigated too. In the chart coordinate $\left(\Omega_{\kappa}, \varphi_{\kappa}\right)$, the Verkley $(1984,1987)$ modons have the form

$\Psi_{\iota}(\lambda, \mu)=\left(\psi_{1} \circ \varphi_{\kappa}^{-1}\right)\left(\lambda^{\prime}, \mu^{\prime}\right)+\left(\psi_{2} \circ \varphi_{\iota}^{-1}\right)(\lambda, \mu)$

To construct the Verkley (1990) uniform modon on the manifold $S^{2}$, it is interpreted as

$\Psi_{\kappa}\left(\lambda^{\prime}, \mu^{\prime}\right)=\left(\psi_{1} \circ \varphi_{\kappa}^{-1}\right)\left(\lambda^{\prime}, \mu^{\prime}\right)+\left(\psi_{2} \circ \varphi_{\iota}^{-1}\right)(\lambda, \mu)$

when the modon center is in the point $\lambda_{o}=270 ; \varphi_{o}=0$. However, to contruct the verkley $(1984,1987,1990)$ with $N^{\prime}$ a point arbitrary on the sphere $S^{2}$ a collection of pairs $\left(\Omega_{\iota}, \varphi_{\iota}\right)(\mathrm{i}>2)$ is needed. Our viewpoint here was to understand the solution of the barotropic vorticity equation on the manifold $S^{2}$ and its use to derive properties of the solutions to the Riemannian manifold $\left(S^{2}, g\right)$.

\section{Acknowledgments}

The author is grateful to A. Aguilar in the preparation of the figures. N. Aguilar, A. Alazraki, and C. Amescua gave valuable suggestions for improving the manuscript.

\section{References}

Abramowitz M. and I. A. Stegun, 1965. Handbook of mathematical functions. Dover, $1046 \mathrm{pp}$.

Aubin T., 1982. Nonlinear analysis on manifolds: Monge-Ampere equations. Springer, New YorkBerlin, 204 pp.

Aubin T., 2001. A course in differential geometry. Graduate Studies in Mathematics, vol. 27. American Mathematical Society, Providence, RI.
Avez A. and Y. Bamberger, 1978. Mouvements sphériques de fluides visqueux incompressibles. J. Mécanique 17, 107-145.

Bihlo A., 2010. Symmetry methods in the atmosphere sciences. Ph.D. thesis. Universitat Wien, $157 \mathrm{pp}$.

Bihlo A., and R. Popovych 2012. Lie reduction and exact solutions of the vorticity equation on the sphere. Physics Letters A 376(14), 1179-1184.

Crum F. X. and D. E. Stevens, 1988. A case study of atmospheric blocking using isentropic analysis. Mon . Wea. Rev. 116, 223-241.

Díaz J. I. and L. Tello, 1999. A nonlinear parabolic problem on a Riemannian manifold without boundary arising in climatology. Collect. Math. 50, 19-51.

Ebin D. and J. Marsden, 1970. Groups of diffeomorphisms and the motion of an incompressible fluid. Ann. Math. 92, 102-163.

Haurwitz B., 1940. The motion of atmospheric disturbances on a spherical Earth. J. Marine Res. 3, 254-267.

Hebey E., 2000. Nonlinear analysis on manifolds: Sobolev spaces and inequalities. Courant Lecure Notes 5. American Mathematical Society, Providence, RI.

Helgason S., 1984. Groups and geometric analysis. Integral geometry, invariant differential operators and spherical functions. Academic Press, Orlando.

Hobson E. W., 1931. The theory of spherical and ellipsoidal harmonics. Chelsea Publishing Company.

Illari L., 1984. A diagnostic study of the potential vorticity in a warm blocking anticyclone. J Atmos. Sci. 41, 3518-3526.

Ilyin A. A., 1991. The Navier-Stokes and Euler equations on two-dimensional closed manifolds. Math. USSR Sbornik 69, 559-579.

Loomis L. and S. Sterberg, 1990. Advanced calculus. Adisson Wesley, 580 pp.

Matsushima Y., 1972. Differentiable manifolds. Pure and applied mathematics. Dekker, 303 pp.

Narcowich F., P. Petrushev and J. Ward, 2006. Decomposition of Besov and Triebel-Lizorkin spaces on the sphere. J. Funct. Anal. 238, 530-564.

Neven E. C., 1992. Quadrupole modons on a sphere. Geophys. Astro. Fluid 65, 105-126.

Pérez-García I. and Y. N. Skiba, 1999. Simulation of exact barotropic vorticity equation solutions using a spectral model. Atmósfera 12, 223-243.

Pérez-García I. and Y. N. Skiba, 2001. Tests of a numerical algorithm for the linear instability study of flows on a sphere. Atmósfera 14, 95-112. 
Pérez-García I., 2001. Análisis de la ecuación de vorticidad barotrópica atmosférica por medio del método espectral. Ph D thesis, UNAM, México, 165 pp.

Pérez-García I., 2014. Rossby-Haurwitz wave perturbation under tropical forcing. Atmósfera 27, 239-249.

Richtmyer R. D., 1981. Principles of advanced mathematical physics, vol. 2. Springer-Verlag.

Rossby C.-G. et al., 1939. Relation between variations in the intensify of the zonal circulation of the atmosphere and the displacement of the semi-permanent centers of action. J. Mar. Res. 2, 38-55.

Simmons A. J., J. M. Wallace and G. W. Branstator, 1983. Barotropic wave propagation and instability, and atmospheric teleconnection patterns. J. Atmos. Sci. 40, 1363-1392.

Skiba Y. N., 1989. Mathematical problems of the dynamics of viscous barotropic fluid on a rotating sphere. Dept. Comput. Mathematics, The USSR Academy of Sciences, Moscow, 178 pp. (English transl.: Indian Institute of Tropical Meteorology, Pune, India, 1990, 211 pp).

Skiba Y. N., 1997. On dimensions of attractive sets of viscous fluids on a sphere under a sphere under qua-periodic forcing. Geophys. Astrophys. Fluid Dyn. 85, 233-242.

Skiba Y. N. and I. Pérez-García, 2006. Testing of a numerical instability study algorithm with the Rossby-Haurwitz waves. Int. Jour. of Appl. Math. 19, 433-460.

Skiba Y. N., and I. Pérez-García, 2009. Numerical spectral method for normal-mode stability study of ideal flows on a rotating sphere. Int. Jour. of Appl. Math. 22, 725-758.
Skiba Y. N., 2012. On the existence and uniqueness of solution to problems of fluid dynamics on a sphere. $J$. Math. Anal. Appl. 388, 622.644.

Stein E. M. and G. Weiss, 1971. Introduction to Fourier analysis on euclidean spaces. Princeton University Press.

Szeptycki P., 1973a. Equations of hydrodynamics on compact Rienmannian manifolds. B. Acad. Pol. Sci. SMAP 21, 335-339.

Szeptycki P., 1973b. Equations of hydrodynamics on manifold diffeomorfic to the sphere. B. Acad. Pol. Sci. SMAP 21, 341-344.

Temam R., 1997. Infinite-dimensional dynamical systems in mechanics and physics. Springer, New York.

Thompson P. D., 1982. A generalized class of exact time-dependent solutions of the vorticity equation for nondivergent barotropic flow. Mon. Wea. Rev. 110, 1321-1324.

Tribbia J. J., 1984. Modons in spherical geometry. Geophys. Astro. Fluid 30, 131-168.

Verkley W. T. M., 1984. The construction of barotropic modons on a sphere. J. Atmos. Sci. 41, 2492-2504.

Verkley W. T. M., 1987. Stationary barotropic modons in westerly background flows. J. Atmos. Sci. 44, 23832398.

Verkley W. T. M., 1990. Modons with uniform absolute vorticity. J. Atmos. Sci. 47, 727-745.

Wu P. and W. T. M. Verkley, 1993. Non-linear structures with multivalued $(q, \Psi)$ relationships - exact solutions of the barotropic vorticity equation on a sphere. Geophys. Astro. Fluid 69, 77-94. 